\title{
Comparative Cost-Effectiveness of Tofacitinib With Continuing Conventional Synthetic Disease- Modifying Anti-Rheumatic Drugs for Active Rheumatoid Arthritis in South Korea
}

\author{
So-Young Ha · Yoon-Bo Shim • Min-Young Lee · Bon-San Koo • \\ Jae-Hoon Kim · Ja-Young Jeon · Hyun-Jeong Yoo • Young-Joo Kim • \\ Ju-Young Shin · Mi-Hai Park (D)
}

Received: October 28, 2020 / Accepted: January 11, 2021 / Published online: January 26, 2021

(C) The Author(s) 2021

\begin{abstract}
Introduction: The objective of this study was to evaluate the cost-effectiveness of initiating treatment with tofacitinib and subsequently incorporating it into a conventional synthetic disease-modifying anti-rheumatic drug (csDMARD) treatment sequence and to compare the cost-effectiveness of this sequence with that of continuing csDMARDs alone in patients with active rheumatoid arthritis (RA).

Methods: A cohort-based Markov model was used to evaluate the cost-effectiveness of two tofacitinib treatment sequences compared with that of continuing the csDMARD treatment sequence over a lifetime. Of the two tofacitinib
\end{abstract}

S.-Y. Ha · Y.-B. Shim · J.-Y. Shin · M.-H. Park ( $ه)$ School of Pharmacy, Sungkyunkwan University, Suwon, Gyeonggi-do, South Korea e-mail: bestway00@skku.edu

M.-Y. Lee

VIAplus, Suwon, Gyeonggi-do, South Korea

B.-S. Koo

Department of Internal Medicine, Inje University Seoul Paik Hospital-Inje University College of

Medicine, Seoul, South Korea

J.-H. Kim

Department of Rheumatology, Korea University

Guro Hospital, Seoul, South Korea

J.-Y. Jeon · H.-J. Yoo · Y.-J. Kim

Pfizer Pharmaceuticals Korea Ltd., Seoul, South

Korea sequences, the first consisted of initial tofacitinib treatment followed by biologic DMARDs (bDMARDs) and the second consisted of csDMARD treatments followed by tofacitinib. A third treatment sequence, continuing the csDMARD treatment sequence before starting bDMARDs, was used as a comparator. Efficacy was assessed using the American College of Rheumatology (ACR) response rates (ACR 20, ACR 50, and ACR 70) after 6 months, which were converted to changes in the health assessment questionnaire-disability index (HAQ-DI) score. Utility was estimated by mapping from the HAQ-DI score, costs were analyzed from a Korean societal perspective, and outcomes were considered in terms of qualityadjusted life-years (QALYs). One-way sensitivity analysis and probabilistic sensitivity analysis were performed to assess the robustness of the model.

Results: The incremental cost-effectiveness ratios over a lifetime for starting with tofacitinib and incorporating tofacitinib into the csDMARD treatment sequence versus continuing csDMARDs only were US $\$ 14,537$ per QALY and US\$7,086 per QALY, respectively. One-way sensitivity analysis and probabilistic sensitivity analysis confirmed the robustness of these results.

Conclusion: Starting with tofacitinib and incorporating it into a csDMARDs treatment sequence is cost-effective compared to continuing csDMARDs alone in patients with RA. 
Keywords: Anti-rheumatic agents; Cost-benefit analysis; Quality-adjusted life-years; Rheumatoid arthritis

\section{Key Summary Points}

\section{Why carry out this study?}

Advanced therapies, such as tofacitinib (TOF), are available for patients with rheumatoid arthritis (RA) who show an inadequate response (IR) to conventional synthetic disease-modifying antirheumatic drugs (csDMARDs), but often these patients continue on csDMARDs alone for a number of reasons.

We have evaluated the cost-effectiveness of starting with TOF and subsequently incorporating this medication into the csDMARD treatment sequence, and compared with that of continuing csDMARDs, in patients with RA who had an IR to csDMARDs.

\section{What was learned from the study?}

The incremental cost-effectiveness ratios (ICERs) over a lifetime for the treatment sequence that started with TOF and then incorporated it into the csDMARD treatment sequence versus continuing csDMARDs were US $\$ 14,537 /$ qualityadjusted life-year (QALY) and US\$7,086 QALY, respectively.

The results show that treatment with TOF was a cost-effective option because the ICERs were lower than the ICER threshold of US\$20,000/QALY.

These results indicate the economic burden and long-term efficacy of TOF and could influence physicians and patients to consider treatment with targeted therapy rather than continuing with csDMARDs.

\section{DIGITAL FEATURES}

This article is published with digital features, including a summary slide, to facilitate understanding of the article. To view digital features for this article go to https://doi.org/10.6084/ m9.figshare.13553621.

\section{INTRODUCTION}

The European League Against Rheumatism (EULAR) recommends that the treatment of rheumatoid arthritis (RA) be initiated with conventional synthetic disease-modifying antirheumatic drugs (csDMARDs) [1]. Should patients fail to respond to csDMARDs, then targeted synthetic DMARDs (tsDMARDs) or biologic DMARDs (bDMARDs) should be included in their therapeutic regimen. In Korea, insurance criteria allow patients who have an inadequate response (IR) to at least two csDMARDs to be reimbursed for the costs of tsDMARDs and bDMARDs. According to the Ministry of Food and Drug Safety, the following medications are available in Korea: the tsDMARDs tofacitinib and baricitinib, which are Janus kinase inhibitors; the bDMARDs adalimumab, etanercept, golimumab, and infliximab, which are tumor necrosis factor inhibitors; abatacept, which is a selective costimulation modulator; tocilizumab, which is an interleukin-6 inhibitor; and rituximab, which is an anti-CD-20 antibody.

Several randomized controlled trials (RCTs) have demonstrated a higher efficacy of tofacitinib [2-4] and bDMARDs [5-8] compared with csDMARDs in patients with moderate to severe RA. However, in real-world clinical practice, patients continue to use csDMARDs for various reasons, including safety concerns, cost considerations, and patient preference $[9,10]$. It is known that the chronic use of csDMARDs can present difficulties for the effective treatment of patients with RA, as it can aggravate symptoms and joint damage [11]. Therefore, initiating tsDMARD or bDMARD treatment at the appropriate time is important to obtain optimal clinical outcomes. 
A recent study conducted in South Korea demonstrated that incorporating tofacitinib into the bDMARD treatment sequence is a costeffective alternative to the bDMARD sequence alone for patients with moderate to severe RA who had an IR to csDMARDs [12]. However, owing to the high cost of these drugs, no study has investigated the cost-effectiveness of tofacitinib versus csDMARDs. Therefore, the objective of this study was to evaluate the costeffectiveness of starting with tofacitinib therapy and subsequently incorporating tofacitinib into the csDMARD treatment sequence in comparison with continuing with csDMARDs alone for the treatment of patients with active RA who had an IR to csDMARDs.

\section{METHODS}

\section{Model Overview}

A cost-utility analysis was performed to compare the cost-effectiveness of two tofacitinib treatment sequences (sequence A: starting with tofacitinib; sequence $\mathrm{C}$ : incorporating tofacitinib into the csDMARD therapeutic sequence) with that of a csDMARDs therapeutic sequence (sequence B: continuing with csDMARDs). A cohort-based Markov model, commonly used to evaluate the cost-effectiveness of treatments, was designed using Microsoft Excel ${ }^{\circledR}$ (Microsoft Corp., Redmond, WA, USA). We modeled a hypothetical cohort of 10,000 patients who had an IR to csDMARDs. Based on the results of a systematic review, we estimated the characteristics of patients using weighted averages of patient characteristics from three selected phase 3 large-scale RCTs [2-4] that had evaluated the efficacy of tofacitinib in patients with RA who had an IR to methotrexate or csDMARDs.

Sequence A, defined as a treatment sequence that started with tofacitinib, was initiated with tofacitinib instead of csDMARDs before the use of bDMARDs and had follow-up bDMARD treatments. Sequence B was defined as a continued csDMARD therapeutic sequence alone and follow-up with bDMARD treatments. Sequence C, defined as a therapeutic sequence with csDMARDs followed by tofacitinib, involved the additional use of tofacitinib after continuing treatment with csDMARDs and follow-up with bDMARD treatments. In all three therapeutic sequences, the follow-up bDMARD treatments comprised adalimumab, tocilizumab, and rituximab in that order. Adalimumab and tocilizumab are the most prescribed tumor and non-tumor necrosis factor inhibitors, respectively, in Korea. Rituximab was selected as the most appropriate drug based on the reimbursement criteria. After all active treatments failed, palliative therapy was maintained until death in each therapeutic sequence (Fig. 1). The cycle length was 6 months based on the followup cycle recommended in the treatment guidelines.

A graphical depiction of the model structure is provided in Fig. 2. The patients were divided into non-responders (American College of Rheumatology response rate $[\mathrm{ACR}]<20$ ) and responders (ACR 20-50, ACR 50-70, or ACR > $70)$ according to the ACR response in the first treatment cycle. In the tofacitinib and bDMARD treatment sequence, non-responders and responders who experienced adverse events (AEs) or lack of efficacy that led to treatment discontinuation were switched to the next treatment in the sequence. In the csDMARD treatment sequence, patients who experienced AEs or lack of efficacy regardless of being a responder or non-responder were switched to the next treatment in the sequence.

The results of this study are presented in terms of the incremental cost-effectiveness ratio (ICER) representing incremental cost per quality-adjusted life-years (QALYs) gained. QALYs and costs were evaluated throughout the lifetime. Cost analysis was performed from a Korean societal perspective, excluding productivity loss costs, using Korean Won (KRW) values as of the third quarter (Q3) of 2019. A discount rate of $5 \%$ was applied to both outcomes and costs.

This is a modeling study using data derived from previously conducted studies and does not contain any new studies involving human participants or animals. 


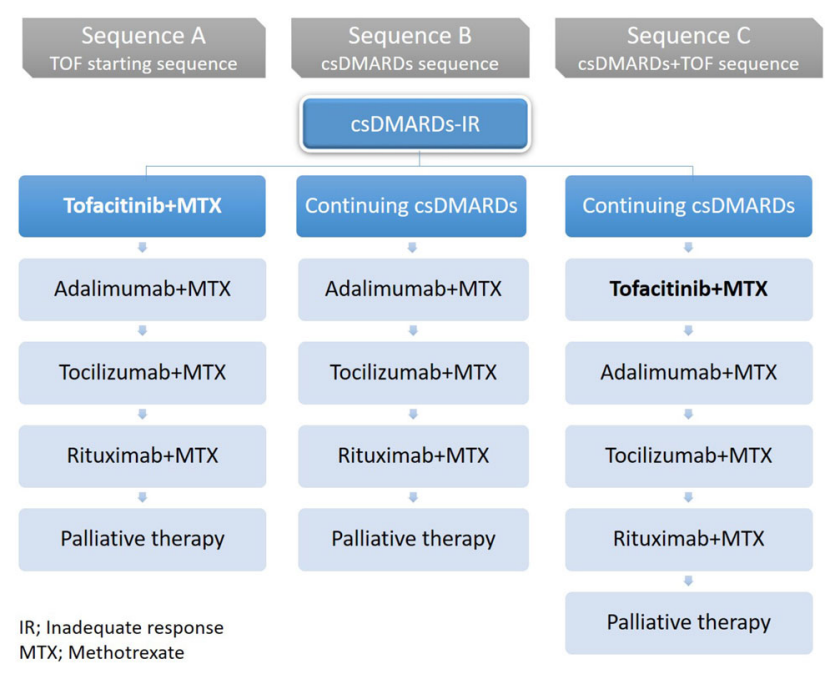

Fig. 1 Simplified treatment strategies used in the model. csDMARDs Conventional synthetic disease-modifying antirheumatic drugs, TOF Tofacitinib

\section{A Start with Tofacitinib (Sequence A)}

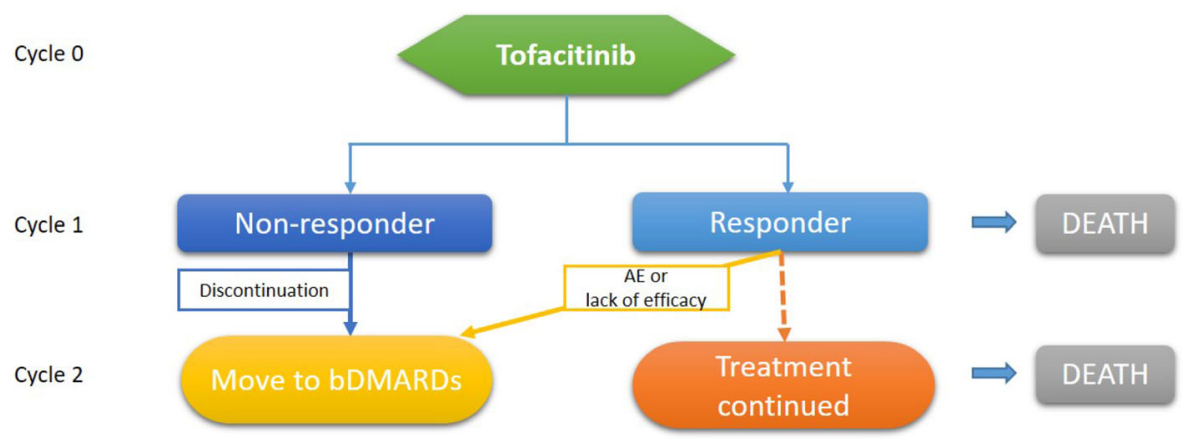

B Continuous use with csDMARDs (Sequence B and C)

Cycle 0

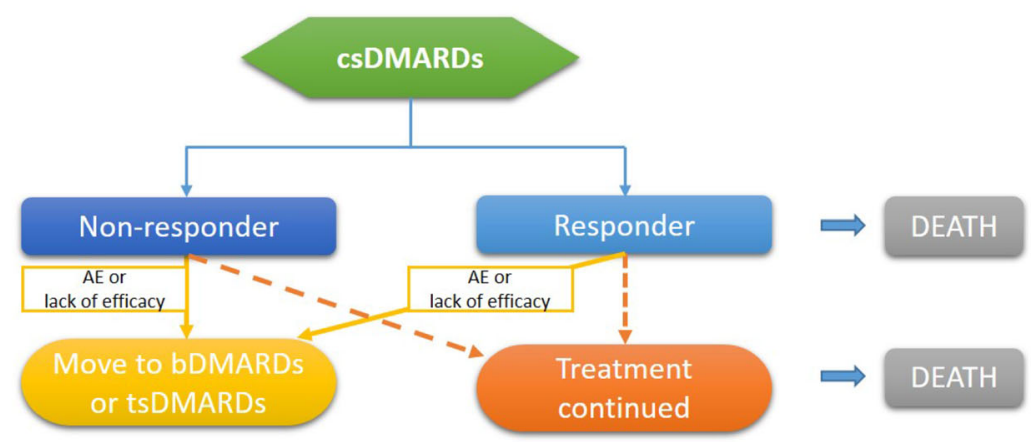

AE; Adverse Event

Fig. 2 Model structures of the treatment sequences. bDMARDS Biologic DMARDs, tsDMARDs targeted synthetic DMARDs 


\section{Efficacy}

The efficacy of each treatment was modeled according to two distinct periods: (1) initial efficacy (at the first 6 months) and (2) subsequent efficacy (after the first 6 months). For initial efficacy, ACR responses in the first 6 months (ACR 20, ACR 50, and ACR 70) were extracted from the data reported in phase 3 RCTs [2-4, 13] and a meta-analysis [14] and then analyzed in the appropriate patient group for each treatment sequence. The ACR response to tofacitinib was calculated using that relative to csDMARDs and relative risk was calculated from the data derived from the meta-analysis of RCTs [2-4]. The changes in the health assessment questionnaire-disability index (HAQ-DI) score were determined based on whether the patients were in the csDMARDs-IR or bDMARDs-IR group and on the ACR response categories (ACR $<20$, ACR 20-50, ACR 50-70, and ACR > 70) [15]. For subsequent efficacy determination, changes in the HAQ-DI score following each treatment per cycle after the first 6 months were estimated according to the annual HAQ-DI changes reported by Stevenson et al. [15]. Patients who discontinued treatment showed no initial effect of the treatment and were moved to the next treatment. All input data are presented in Table 1.

\section{Probability of Discontinuation}

Non-responders $(A C R<20)$ to tofacitinib or bDMARDs determined after each cycle were withdrawn from the treatment and switched to the next follow-up treatment. Patients receiving csDMARDs continued the same treatment until discontinuation owing to AEs or lack of efficacy regardless of the ACR response. In subsequent cycles, the probability of discontinuing each treatment was estimated using the results of long-term observational studies to determine the probability of all-cause discontinuation [16-18]. These studies had sufficient long-term data to use the probability of discontinuation: 5 years for tofacitinib [16], 20 months for csDMARDs [17], and 6 years for bDMARDs [18]. The probability of discontinuation per cycle was converted from the long-term probability of drug survival using the following formula:

$$
P_{\mathrm{d}}=1-P_{\mathrm{ds}}^{1 / t} .
$$

where $P_{\mathrm{d}}$ is the probability of discontinuation per cycle, $P_{\mathrm{ds}}$ is the probability of drug survival based on observational studies, and $t$ is the continuation period derived from observational studies divided by the 1-cycle duration (Table 1).

\section{Mortality}

The mortality of patients with RA was calculated using the HAQ-DI scores and the general population mortality rate (adjusted mortality = general mortality $\times 1.33^{(\mathrm{HAQ}-\mathrm{DI})}$ [19]. The general population mortality was extracted from age- and sex-specific complete life tables for the Korean population.

\section{Health-Related Quality of Life}

The health-related quality of life of the patient cohort for each cycle and treatment was calculated by mapping the HAQ-DI scores onto the EuroQoL5D (EQ-5D) utility values. The mapping equation from a previous tofacitinib study, which developed a linear regression model $(\mathrm{EQ}-5 \mathrm{D}=$

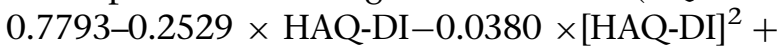
$0.0013 \times$ age $+0.0310 \times$ female $+0.0010 \times[\mathrm{RA}$ duration year]), was used to predict the EQ-5D scores [12].

\section{Cost}

All costs were adjusted to the Q3 2019 USD value using the Q3 2019 exchange rate (1 $\mathrm{USD}=1193.947 \mathrm{KRW})$. Direct medical costs included medication, administration, monitoring costs, and doctors' fees. Direct non-medical costs, including travel expenses and costs of auxiliary device, dietary supplement, and home assistance, were calculated based on a previous cost-of-illness study [20]. The cost of illness was defined as the value of resources expended or resources foregone as a result of the health 
Table 1 Characteristics of the model and model input parameters

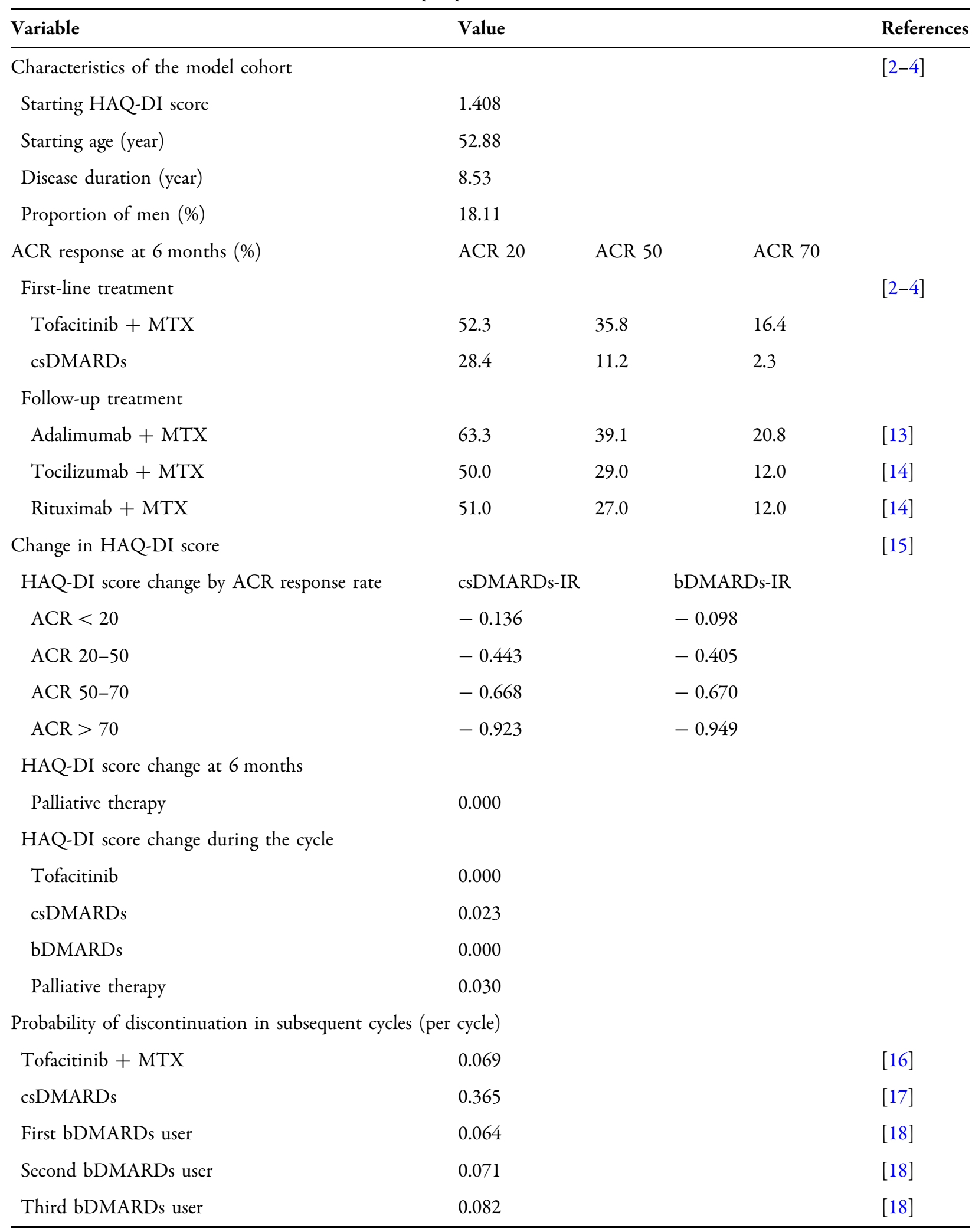


Table 1 continued

\begin{tabular}{lll}
\hline Variable & Value & References \\
\hline Mortality risk for patients with RA per HAQ-DI & 1.33 & {$[19]$} \\
\hline
\end{tabular}

ACR American College of Rheumatology measure of improvement, $b D M A R D s$ biologic disease-modifying anti-rheumatic drugs, csDMARDs conventional synthetic disease-modifying anti-rheumatic drugs, HAQ-DI Health Assessment Questionnaire-Disability Index, $M T X$ methotrexate, $R A$ rheumatoid arthritis

problem. Cost was categorized into that incurred during the first treatment cycle and that incurred during subsequent cycles because of the difference in the monitoring period, as recommended by the reimbursement criteria of the Health Insurance Review \& Assessment Service.

Medication costs were calculated using the listed drug prices and treatment schedules. The costs of csDMARDs were calculated taking into consideration the most popular csDMARDs used in clinical trials, such as methotrexate, leflunomide, hydroxychloroquine, and sulfasalazine [3]. The treatment schedule was based on the dosing schedules recommended by the Korean Ministry of Food and Drug Safety: $5 \mathrm{mg}$ twice daily for oral tofacitinib; $40 \mathrm{mg}$ once every 2 weeks for subcutaneous adalimumab; $162 \mathrm{mg}$ once every 2 weeks for subcutaneous tocilizumab; and $1000 \mathrm{mg}$ twice, 2 weeks apart, every 24 weeks for intravenous rituximab. Tofacitinib and bDMARD treatments were combined with methotrexate, which is a basic medicine used in the treatment of RA.

Administration costs for injections were dependent on the method of administration. For every intravenous injection, such as that of rituximab, the patient is required visit the hospital. For subcutaneous injections, such as that of adalimumab and tocilizumab, the patient is required visit the hospital for the first injection and subsequently to self-inject at home. Pharmacy dispensing costs for oral drugs were calculated based on the assumption that oral drugs were prescribed at every visit.

Monitoring costs were calculated using the number of visits according to the ACR guideline, and doctors' fees were calculated based on the assumption that patients were seen by the doctor at every hospital visit for administration and monitoring.
The costs of illness were calculated using the three RA functional status classes (class 1: HAQ score 0-0.75; class 2: HAQ score 0.75-1.50; combined class 3-4: HAQ score 1.50-3.0) to reflect the deteriorating health condition of patients. The cost was estimated using the regression coefficients for each class obtained through multiple regression analysis. For the active treatment, direct medical costs, including those for private physiotherapy, traditional Chinese medicine, and other alternative medicines, as well as direct non-medical costs, including those for travel, auxiliary devices, dietary supplements, and home assistance, were assessed. The total direct costs also took into account the cost of illness, which included palliative therapy costs (Table 2). Total costs according to medications used are shown in Table 3.

\section{Sensitivity Analysis}

To verify the validity of the assumptions used in the model, one-way sensitivity and probabilistic

Table 2 Cost of illness by functional status

\begin{tabular}{lcll}
\hline $\begin{array}{l}\text { Functional } \\
\text { status }\end{array}$ & $\begin{array}{l}\text { HAQ-DI } \\
\text { score } \\
\text { range }\end{array}$ & $\begin{array}{l}\text { Estimated } \\
\text { costs during } \\
\text { active } \\
\text { treatment } \\
\text { (USD) }\end{array}$ & $\begin{array}{l}\text { Estimated } \\
\text { costs during } \\
\text { palliative } \\
\text { treatment } \\
\text { (USD) }\end{array}$ \\
\hline Class 1 & $0-0.75$ & 335 & 1081 \\
Class 2 & $0.75-1.5$ & 422 & 1361 \\
Class 3-4 & $1.5-3.0$ & 607 & 1958 \\
\hline USD U.S. dollar (1 USD $=1193.947$ & Korean Won \\
(KRW) (exchange rate for the third quarter of 2019)
\end{tabular}


Table 3 Total cost per cycle by medication

\begin{tabular}{lll}
\hline Variable & \multicolumn{2}{l}{ Estimates (USD) } \\
\cline { 2 - 3 } First & $\begin{array}{l}\text { Subsequent } \\
\text { cycles }\end{array}$ \\
\hline cycle & & \\
Medication costs & 3347 & 3347 \\
csDacitinib + MTX & 104 & 98 \\
Adalimumab + MTX & 4152 & 4152 \\
Tocilizumab + MTX & 3650 & 3650 \\
Rituximab + MTX & 3495 & 3495 \\
Doctor's fee & & \\
Tofacitinib + MTX & 71 & 24 \\
csDMARDs & 48 & 24 \\
Adalimumab + MTX & 71 & 24 \\
Tocilizumab + MTX & 71 & 24 \\
Rituximab + MTX & 36 & 36 \\
Administration and & & 81 \\
dispensing costs & & \\
Tofacitinib + MTX & 58 & 26 \\
csDMARDs & 33 & 21 \\
Adalimumab + MTX & 34 & 15 \\
Tocilizumab + MTX & 34 & 15 \\
Rituximab + MTX & 36 & 36 \\
Monitoring costs & & \\
Tofacitinib + MTX & 81 & 81 \\
csDMARDs & 158 & 81 \\
Adalimumab + MTX & 81 & 81 \\
Tocilizumab + MTX & 81 & 81 \\
Rituximab + MTX & 81 & 81 \\
\hline & & \\
\hline
\end{tabular}

sensitivity analyses were performed. For oneway sensitivity analysis, we considered the relative risk of death, the HAQ-utility relation equation, cost of illness, time horizon, and discount rate. We ran 10,000 iterations of the decision model in a Monte Carlo simulation for the probabilistic sensitivity analysis. The relative risk of tofacitinib and csDMARDs ACR responses, relative risk of death, ACR response, probability of discontinuation, and costs were included in the analysis. The applied function had a log-normal distribution for relative risk, a beta distribution for the ACR response and probability of discontinuation, and a gamma distribution for cost. The data are expressed as the mean and standard errors (SE). If no values were available for indicated parameters, the standard errors were assumed to be $25 \%$ of the mean.

\section{RESULTS}

\section{Base Case Analysis}

The results of the base case analysis for each treatment sequence are shown in Table 4 . For sequence $B$, in which patients continuously used csDMARDs despite the feasibility of bDMARDs or tsDMARDs, the total cost per patient throughout their lifetime was US\$92,137, and the QALY gained per patient was 7.45. Sequence A, which involved starting with tofacitinib, resulted in higher costs (US\$104,054) and greater gains in QALYs per patient (8.27 QALYs). Therefore, the ICER with sequence A was estimated to be US\$14,537 per QALY gained compared with sequence B. For sequence $\mathrm{C}$, in which csDMARDs were followed by tofacitinib, the total cost per patient was US\$97,406 and the QALY gained per patient was 8.19. Thus, the ICER with sequence $C$ was estimated to be US\$7086.00 per QALY gained compared with sequence $B$.

\section{Sensitivity Analysis}

The results of the one-way sensitivity analysis are described in Table 5 and reveal the effect of varying one parameter in the model at a time. Regarding sequence A versus $B$, mapping the HAQ-DI equation was the most sensitive tool, and the application of the equations reported in other studies [21-24] resulted in an increase in the ICER from $8.35 \%$ in the base case to $19.70 \%$. 
Table 4 Results of the base case analysis

\begin{tabular}{|c|c|c|c|c|}
\hline \multirow[t]{2}{*}{ Variable } & \multicolumn{2}{|l|}{ Result 1} & \multicolumn{2}{|l|}{ Result 2} \\
\hline & $\begin{array}{l}\text { Treatment } \\
\text { Sequence } A^{a}\end{array}$ & $\begin{array}{l}\text { Comparator } \\
\text { Sequence } \mathbf{B}^{\mathbf{a}}\end{array}$ & $\begin{array}{l}\text { Treatment } \\
\text { Sequence } C^{a}\end{array}$ & $\begin{array}{l}\text { Comparator } \\
\text { Sequence } B^{\mathbf{a}}\end{array}$ \\
\hline \multicolumn{5}{|l|}{ Model results } \\
\hline Total costs $\left(\mathrm{USD}^{\mathrm{b}}\right)$ & 104,054 & 92,137 & 97,406 & 92,137 \\
\hline Effectiveness (QALY) & 8.27 & 7.45 & 8.19 & 7.45 \\
\hline \multicolumn{5}{|l|}{ Incremental results } \\
\hline Total costs (USD) & 11,918 & & 5269 & \\
\hline Effectiveness (QA LY) & 0.82 & & 0.74 & \\
\hline ICER (USD/QALY) & 14,537 & & 7086 & \\
\hline
\end{tabular}

ICER incremental cost-effectiveness ratio, QALY quality-adjusted life-year

a Sequence A: Treatment sequence started with tofacitinib instead of csDMARDs followed by bDMARDs. Sequence B: Started and continuing use of csDMARD alone and follow-up with bDMARDs. Sequence C: Treatment sequence started with csDMARDs followed by the addition of tofacitinib to treatment regimen (continued use of csDMARDs) and followup with bDMARDs

b 1 USD $=1193.947$ KRW (exchange rate for the third quarter of 2019)

The ICER increased with an adjustment for the relative risk of mortality (2.73) reported in another study [25]; however, it decreased with an adjustment for no risk of mortality. When the cost of illness was calculated using the unadjusted costs classified according to the RA functional status class, the ICER increased by $8.16 \%$ from the base case. For sequence $C$ versus $\mathrm{B}$, the result followed the same trend as that for sequence A versus B: the ICER increased with the application of a higher relative risk of mortality, other mapping equations of HAQ-DI [21-24], and the cost of illness calculated from the raw data.

The results of the probabilistic sensitivity analysis iterated 10,000 times are presented using cost-effectiveness acceptability curves in Fig. 3. Sequences A and C had a 98.1 and 100\% likelihood of being cost-effective, respectively, compared with sequence $B$, at a threshold of US\$20,000 per QALY.

\section{DISCUSSION}

We have developed a cohort-based Markov model to simulate the cost-effectiveness of two tofacitinib treatment sequences and compared this with the cost-effectiveness of a treatment sequence of continued csDMARDs alone. The ICERs for the treatment sequence starting with tofacitinib and subsequently incorporating tofacitinib into the csDMARD treatment sequence versus the treatment sequence of continuing the use of csDMARDs were US\$14,537 per QALY gained and US\$7,086 per QALY gained, respectively, over a lifetime. In our analysis, the ICERs were lower than the ICER threshold determined based on the commonly cited global cost-effectiveness threshold of US\$20,000 per QALY gained. Therefore, we can conclude that treatment with tofacitinib is cost-effective from a Korean societal perspective. The sensitivity analysis confirmed that the results were robust.

Some patients tend to continue using csDMARDs despite an IR to this treatment. According to the nationwide Korean Biologics Registry [26], the number of prior non-biologic DMARDs recorded for first-time users of bDMARDs in Korea is 3.06. According to the reimbursement criteria for tsDMARDs or bDMARDs, the payer can cover the cost of these drugs for patients who have had a prior IR to 


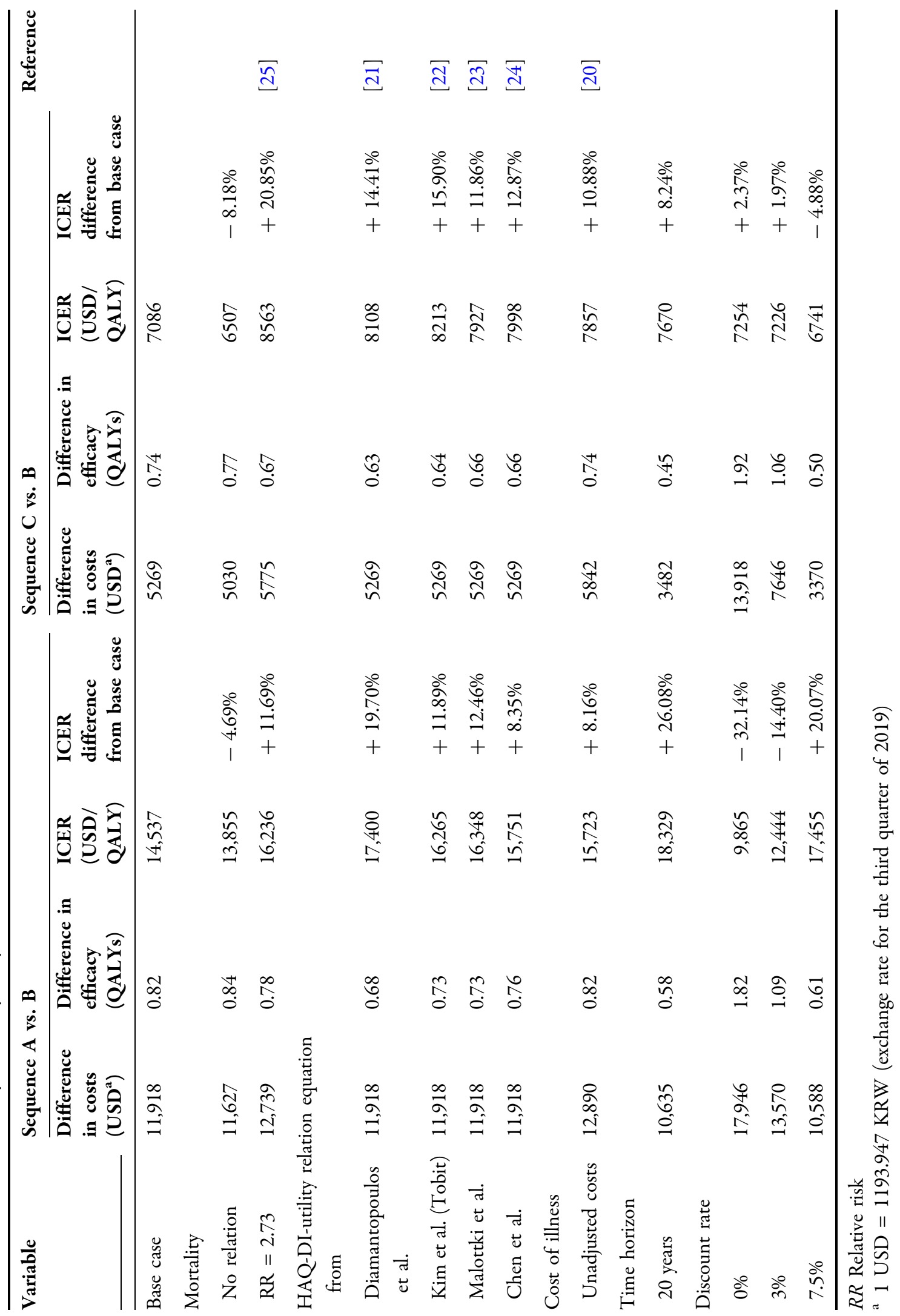




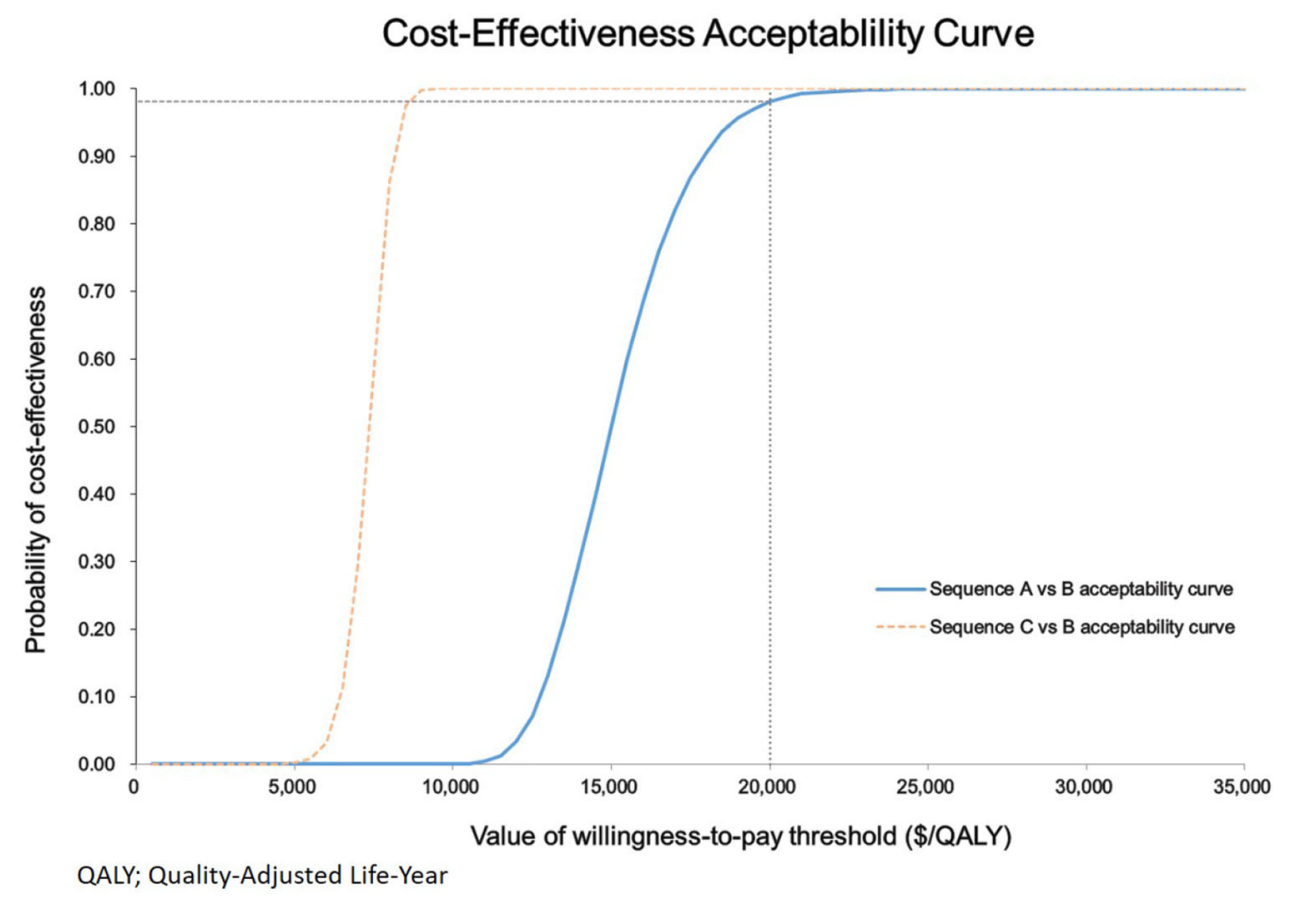

Fig. 3 Cost-effectiveness acceptability curves of the two results obtained. See text and footnote of Table 4 for explanation of treatment sequences

more than two csDMARDs; however, some patients still tend remain on csDMARD treatment. Patients who are eligible for treatment with tsDMARDs or bDMARDs continue receiving csDMARDs for several reasons. For example, some physicians are concerned about the safety profiles associated with tsDMARDs or bDMARDs, such as serious infections and malignancies [27]; therefore, they tend to continue the traditional csDMARD treatments that patients have been using. From a patient's perspective, many patients prefer the orally administered csDMARDs over either injected or infused bDMARDs and are more likely to comply with the administration regimen of an oral drug [28]. Moreover, the cost of tsDMARDs or bDMARDs is much higher than that of csDMARDs. As patients with RA require longterm treatment, even life-long treatment, to control their chronic disease, a high drug cost is one of the most important contributing factors to their overall disease burden [29].

Despite these reasons, appropriate treatment is important to delay disease progression as assessed by the HAQ-DI score. Appropriate treatment influences both costs and QALYs via a reduction in the use of resources, such as joint replacements and hospitalizations, and the prevention of a deterioration in the health status [30]. Patients treated with a targeted therapy or bDMARDs achieve a higher remission rate than patients who continue treatment with csDMARDs [10, 31], and the former treatments are more beneficial than csDMARDs with respect to controlling disease exacerbation [32-35]. Although patients also continue treatment with csDMARDs for other reasons, tofacitinib might play an important role in the initiation of advanced therapy as it is an oral treatment that may improve patient compliance and reduce long-term medical costs and socioeconomic impacts [10].

Many previous studies on tofacitinib have not investigated its cost-effectiveness versus that of csDMARDs because the drug is expensive; however, our study examined the cost-effectiveness utilizing the costs of illness calculated using the three-stage RA functional status class of the HAQ-DI to consider the additional costs of deteriorating health 
conditions for patients with a poor response. The recent drop in the prices of tsDMARDs and bDMARDs also contributed to these results. Moreover, these cost-effectiveness results were obtainable because of the unique reimbursement criteria in Korea wherein only patients who previously experienced treatment failure with at least two csDMARDs are covered. As demonstrated in this analysis, the treatment sequence starting with tofacitinib is a cost-effective option compared with that of csDMARDs alone. Therefore, our results reveal the economic burden and long-term efficacy of these treatment sequences in patients and can be used to encourage clinicians and patients to consider treatment with targeted therapies rather than continuing with csDMARDs. Treatment with an appropriate drug, which should be clinically and radiologically effective, is crucial to control RA via the suppression of joint damage progression [11].

This cost-effectiveness study has several limitations. First, there were a few limitations to the available data. Because of the insufficient clinical trial data available for adalimumab administration after tofacitinib administration, we considered the data from RCTs of patients with an IR to methotrexate as the data for patients receiving the csDMARD treatment sequence. Additionally, the inadequate ACR response to csDMARDs was reflected in patients continuing csDMARDs because if a patient was switched to another csDMARDs following lack of efficacy to one csDMARD, the resulting changes in ACR response could not be replicated. Moreover, insufficient data may have led to the inclusion of some bias in our analysis; however, clinically valid assumptions were incorporated into the model to reduce the uncertainty of results. The assumptions that the efficacy of adalimumab after tofacitinib is similar to that after csDMARDs and that the ACR response to csDMARDs does not aggravate after a lack of efficacy with csDMARDs are the most conservative reasons for recommending the use of tofacitinib. Second, the EULAR response is commonly used to diagnose the severity of RA in clinical practice and to define the reimbursement criteria for bDMARDs in Korea. However, as there are insufficient RCTs to accurately estimate patient outcomes according to the EULAR response, we used the ACR response to classify the efficacy of treatment. If sufficient EULAR response data are available in the future, cost-effectiveness considering the EULAR response data would also be meaningful. Lastly, it would be difficult to generalize these results to other countries. The reimbursement criteria and drug price policy vary among countries; therefore, these results are unique to the Korean healthcare system and the societal costs are derived from a Korean perspective. Further research indicating costs in other countries would provide a basis for meaningful comparisons with our findings.

\section{CONCLUSIONS}

This cost-effectiveness analysis from a Korean societal perspective showed that the treatment sequence starting with tofacitinib is more costeffective than continuing csDMARDs alone. Incorporating tofacitinib into the csDMARD treatment sequence is also more cost-effective than continuing csDMARDs for patients with moderate to severe RA. These results, reflecting the additional costs associated with deteriorating health conditions, support the importance of using appropriate targeted therapies.

\section{ACKNOWLEDGEMENTS}

Funding. This research was sponsored by Pfizer Pharmaceuticals Korea Ltd. The sponsor funded the journal's Rapid Service fee and was involved in the design of the study and writing of the manuscript but had no involvement in the management and interpretation of the data.

Editorial Assistance. We would like to thank Editage for English language editing, the funding of which was funded by Sungkyunkwan University.

Authorship. All named authors meet the International Committee of Medical Journal Editors (ICMJE) criteria for authorship for this 
manuscript, take responsibility for the integrity of the work as a whole, and have given their approval for this version to be published.

Authorship Contributions. So-Young Ha, Yoon-Bo Sim, Min-Young Lee, Ju-Young Shin, and Mi-Hai contributed to the study design. SoYoung Ha, Yoon-Bo Sim, and Min-Young Lee contributed to data analysis. Bon-San Koo and Jae-Hoon Kim provided medical advice to reflect real-world clinical practice. Ja-Young Jeon, Hyun-Jeong Yoo, and Young-Joo Kim provided technical assistance. All authors had full access to all models in this study and take complete responsibility for the integrity of the model and accuracy of the data analysis; they all contributed to data interpretation and planning and review of the manuscript content.

Disclosures. Ja-Young Jeon, Hyun-Jeong Yoo, and Young-Joo Kim are employees of Pfizer Pharmaceuticals Korea Ltd. So-Young Ha, YoonBo Sim, Min-Young Lee, Bon-San Koo, Jae-Hoon Kim, Ju-Young Shin, and Mi-Hai Park have nothing to disclose.

Compliance with Ethics Guidelines. This article is a modeling study using data derived from previously conducted studies and does not contain any new studies with human participants or animals performed by any of the authors.

Data Availability. The datasets generated during and/or analyzed during the current study are available from the corresponding author on reasonable request.

Open Access. This article is licensed under a Creative Commons Attribution-NonCommercial 4.0 International License, which permits any non-commercial use, sharing, adaptation, distribution and reproduction in any medium or format, as long as you give appropriate credit to the original author(s) and the source, provide a link to the Creative Commons licence, and indicate if changes were made. The images or other third party material in this article are included in the article's Creative Commons licence, unless indicated otherwise in a credit line to the material. If material is not included in the article's Creative Commons licence and your intended use is not permitted by statutory regulation or exceeds the permitted use, you will need to obtain permission directly from the copyright holder. To view a copy of this licence, visit http://creativecommons.org/licenses/by$\mathrm{nc} / 4.0 /$.

\section{REFERENCES}

1. Smolen JS, Landewé RBM, Bijlsma JWJ, et al. EULAR recommendations for the management of rheumatoid arthritis with synthetic and biological disease-modifying antirheumatic drugs: 2019 update. Ann Rheum Dis. 2020;79(6):685-99.

2. van Vollenhoven RF, Fleischmann R, Cohen S, et al. Tofacitinib or adalimumab versus placebo in rheumatoid arthritis. N Engl J Med. 2012;367(6): 508-19.

3. Kremer J, Li ZG, Hall S, et al. Tofacitinib in combination with nonbiologic disease-modifying antirheumatic drugs in patients with active rheumatoid arthritis: a randomized trial. Ann Intern Med. 2013;159(4):253-61.

4. van der Heijde D, Tanaka Y, Fleischmann R, et al. Tofacitinib (CP-690,550) in patients with rheumatoid arthritis receiving methotrexate: twelve-month data from a twenty-four-month phase III randomized radiographic study. Arthritis Rheum. 2013;65(3):559-70.

5. Muszbek N, Proudfoot C, Fournier M, et al. Economic evaluation of sarilumab in the treatment of adult patients with moderately-to-severely active rheumatoid arthritis who have an inadequate response to conventional synthetic disease-modifying antirheumatic drugs. Adv Ther. 2019;36(6): 1337-57.

6. Whittington MD, McQueen RB, Ollendorf DA, et al. Assessing the value of sarilumab monotherapy for adults with moderately to severely active rheumatoid arthritis: a cost-effectiveness analysis. J Managed Care Specialty Pharm. 2019;25(1):80-7.

7. Joensuu JT, Aaltonen KJ, Aronen P, et al. Cost-effectiveness of biologic compared with conventional synthetic disease-modifying anti-rheumatic drugs in patients with rheumatoid arthritis: a Register study. Rheumatology (Oxford). 2016;55(10): 1803-11. 
8. Eriksson JK, Karlsson JA, Bratt J, et al. Cost-effectiveness of infliximab versus conventional combination treatment in methotrexate-refractory early rheumatoid arthritis: 2-year results of the registerenriched randomised controlled SWEFOT trial. Ann Rheum Dis. 2015;74(6):1094-101.

9. Hsiao B, Fraenkel L. Patient preferences for rheumatoid arthritis treatment. Curr Opin Rheumatol. 2019;31(3):256-63.

10. Song JJ, Song YW, Bae SC, et al. Treat-to-target strategy for asian patients with early rheumatoid arthritis: result of a multicenter trial in Korea. J Korean Med Sci. 2018;33(52):e346.

11. van der Kooij SM, de Vries-Bouwstra JK, GoekoopRuiterman YP, et al. Limited efficacy of conventional DMARDs after initial methotrexate failure in patients with recent onset rheumatoid arthritis treated according to the disease activity score. Ann Rheum Dis. 2007;66(10):1356-62.

12. Lee MY, Park SK, Park SY, et al. Cost-effectiveness of tofacitinib in the treatment of moderate to severe rheumatoid arthritis in South Korea. Clin Ther. 2015;37(8):1662-76.e2.

13. Keystone EC, Kavanaugh AF, Sharp JT, et al. Radiographic, clinical, and functional outcomes of treatment with adalimumab (a human anti-tumor necrosis factor monoclonal antibody) in patients with active rheumatoid arthritis receiving concomitant methotrexate therapy: a randomized, placebo-controlled, 52-week trial. Arthritis Rheum. 2004;50(5):1400-11.

14. Vieira MC, Zwillich SH, Jansen JP, Smiechowski B, Spurden D, Wallenstein GV. Tofacitinib versus biologic treatments in patients with active rheumatoid arthritis who have had an inadequate response to tumor necrosis factor inhibitors: results from a network meta-analysis. Clin Ther. 2016;38(12):2628-41.e5.

15. Stevenson M, Archer R, Tosh J, et al. Adalimumab, etanercept, infliximab, certolizumab pegol, golimumab, tocilizumab and abatacept for the treatment of rheumatoid arthritis not previously treated with disease-modifying antirheumatic drugs and after the failure of conventional disease-modifying antirheumatic drugs only: systematic review and economic evaluation. Health Technol Assess. 2016;20(35):1-610.

16. Wollenhaupt J, Lee EB, Curtis JR, et al. Safety and efficacy of tofacitinib for up to 95 years in the treatment of rheumatoid arthritis: final results of a global, open-label, long-term extension study. Arthritis Res Ther. 2019;21(1):89.
17. Geborek P, Crnkic M, Petersson IF, Saxne T. Etanercept, infliximab, and leflunomide in established rheumatoid arthritis: clinical experience using a structured follow up programme in southern Sweden. Ann Rheum Dis. 2002;61(9):793-8.

18. Kondo M, Yamada H. Drug survival rates of biological disease-modifying antirheumatic drugs and Janus kinase-inhibitor therapy in 801 rheumatoid arthritis patients: a 14 year-retrospective study from a rheumatology clinic in Japan. Modern Rheumatol. 2018;2018:1-19.

19. Wolfe F, Mitchell DM, Sibley JT, et al. The mortality of rheumatoid arthritis. Arthritis Rheum. 1994;37(4):481-94.

20. Lee TJ, Park BH, Son HK, et al. Cost of illness and quality of life of patients with rheumatoid arthritis in South Korea. Value Health. 2012;15(1 Suppl): S43-9.

21. Diamantopoulos A, Benucci M, Capri S, et al. Economic evaluation of tocilizumab combination in the treatment of moderate-to-severe rheumatoid arthritis in Italy. J Med Econ. 2012;15(3):576-85.

22. Kim HL, Kim D, Jang EJ, et al. Mapping health assessment questionnaire disability index (HAQ-DI) score, pain visual analog scale (VAS), and disease activity score in 28 joints (DAS28) onto the EuroQol-5D (EQ-5D) utility score with the KORean Observational study Network for Arthritis (KORONA) registry data. Rheumatol Int. 2016;36(4): 505-13.

23. Malottki K, Barton P, Tsourapas A, et al. Adalimumab, etanercept, infliximab, rituximab and abatacept for the treatment of rheumatoid arthritis after the failure of a tumour necrosis factor inhibitor: a systematic review and economic evaluation. Health Technol Assess. 2011;15(14):1-278.

24. Chen YF, Jobanputra P, Barton P, et al. A systematic review of the effectiveness of adalimumab, etanercept and infliximab for the treatment of rheumatoid arthritis in adults and an economic evaluation of their cost-effectiveness. Health Technol Assess. 2006;10(42):iii-iv, xi-xiii, 1-229.

25. Sokka T, Hakkinen A, Krishnan E, Hannonen P. Similar prediction of mortality by the health assessment questionnaire in patients with rheumatoid arthritis and the general population. Ann Rheum Dis. 2004;63(5):494-7.

26. Park DJ, Choi SJ, Shin K, et al. Switching profiles in a population-based cohort of rheumatoid arthritis receiving biologic therapy: results from the KOBIO registry. Clin Rheumatol. 2017;36(5):1013-22. 
27. Ramiro S, Sepriano A, Chatzidionysiou K, et al. Safety of synthetic and biological DMARDs: a systematic literature review informing the 2016 update of the EULAR recommendations for management of rheumatoid arthritis. Ann Rheum Dis. 2017;76(6):1101-36.

28. Alten R, Kruger K, Rellecke J, et al. Examining patient preferences in the treatment of rheumatoid arthritis using a discrete-choice approach. Patient Prefer Adherence. 2016;10:2217-28.

29. Strand V, Khanna D. The impact of rheumatoid arthritis and treatment on patients' lives. Clin Exp Rheumatol. 2010;28(3 Suppl 59):S32-40.

30. Finckh A, Bansback N, Marra CA, et al. Treatment of very early rheumatoid arthritis with symptomatic therapy, disease-modifying antirheumatic drugs, or biologic agents: a cost-effectiveness analysis. Ann Intern Med. 2009;151(9):612-21.

31. Sung YK, Cho SK, Kim D, et al. Comparative effectiveness of treatment options after conventional DMARDs failure in rheumatoid arthritis. Rheumatol Int. 2017;37(6):975-82.

32. Weinblatt ME, Keystone EC, Furst DE, et al. Adalimumab, a fully human anti-tumor necrosis factor alpha monoclonal antibody, for the treatment of rheumatoid arthritis in patients taking concomitant methotrexate: the ARMADA trial. Arthritis Rheum. 2003;48(1):35-45.

33. Keystone EC, Schiff MH, Kremer JM, et al. Onceweekly administration of $50 \mathrm{mg}$ etanercept in patients with active rheumatoid arthritis: results of a multicenter, randomized, double-blind, placebocontrolled trial. Arthritis Rheum. 2004;50(2): 353-63.

34. Smolen JS, Kay J, Doyle M, et al. Golimumab in patients with active rheumatoid arthritis after treatment with tumor necrosis factor alpha inhibitors: findings with up to five years of treatment in the multicenter, randomized, double-blind, placebo-controlled, phase 3 GO-AFTER study. Arthritis Res Therapy. 2015;17:14.

35. Schiff M, Keiserman M, Codding C, et al. Efficacy and safety of abatacept or infliximab vs placebo in ATTEST: a phase III, multi-centre, randomised, double-blind, placebo-controlled study in patients with rheumatoid arthritis and an inadequate response to methotrexate. Ann Rheum Dis. 2008;67(8):1096-103. 